\title{
ELEMENTOS CIRCUNDANTES A LOS COMITÉS DE ÉTICA QUE DIFICULTAN SU RAZONAMIENTO DELIBERATIVO
}

\author{
Liliana Mondragón Barrios ${ }^{1}$
}

Resumen: Los comités de ética se encuentran entre las manifestaciones más visibles de la bioética. La aceptación que han recibido estas instancias en los últimos años ha sido amplia, pero poco se puede decir acerca de sus funciones y sus metas, principalmente porque se ha rezagado el estudio del trabajo al interior de los comités. El objetivo de este manuscrito es analizar los elementos circunscritos al proceso interno de los comités de ética que están dificultando su razonamiento deliberativo. Elementos como la ausencia de deliberación desde el saber ético, la tensión entre el marco ético y el legal, las particularidades del campo de la salud dentro del discernimiento ético y la consideración de los comités como grupos, deben reconocerse y discutirse en forma permanente y crítica como trabajo autorregulativo de los comités para, de esta manera, conseguir el consenso social e institucional.

Palabras clave: comités de ética en investigación, ética, aspectos bioéticos

\section{Elements surrounding the ethics committees that hinder their deliberative reasoning}

Abstract: Ethics committees are among the most visible manifestations of bioethics. The acceptance these instances have received in recent years has been extensive, but little can be said about their roles and goals, mainly because the analysis of what happens inside the committees has been lagged behind. The aim of this paper is to analyze the elements involved in the inner processes of ethical committees, which are complicating their deliberative reasoning. Elements such as the absence of deliberation from ethical knowledge, the tension between the ethical and legal frameworks, the particularities of the health care field within ethical discernment and the consideration of committees as groups should be permanently and critically recognized and discussed, as self-regulatory work by committees, in order to achieve social and institutional consensus.

Key words: research ethics committees, ethics, bioethics issues

\section{Elementos circundantes aos comitês de ética que dificultam a sua fundamentaçáo deliberativa}

Resumo: Os comitês de ética se encontram entre as manifestações mais visíveis da bioética. A aceitação que tiveram estas instâncias nos últimos anos foi ampla, porém pouco se pode dizer acerca de suas funçôes e metas, principalmente porque se tem postergado o estudo do trabalho no interior dos comitês. O objetivo deste manuscrito é analisar os elementos circunscritos ao processo interno dos comitês de ética que estão dificultando a sua fundamentação deliberativa. Elementos como a ausência de deliberação a partir do saber ético, a tensão entre o marco ético e o legal, as particularidades do campo da saúde dentro do discernimento ético e a consideraçáo dos comitês como grupos, devem ser reconhecidos e discutidos de forma permanente e crítica como trabalho autorregulativo dos comitês para, desta maneira, conseguir o consenso social e institucional.

Palavras-chave: comitês de ética em investigação, ética, aspectos bioéticos

1 Instituto Nacional de Psiquiatría Ramón de la Fuente Muñiz, México

Correspondencia: lilian@imp.edu.mx 


\section{Introducción}

El comité encomendado al abordaje ético de los problemas que se suscitan en el ámbito de la investigación es un espacio de deliberación y de educación(1), cuya función dialógica opera con pluralidad de visiones y opiniones. Promueve la reflexión alejada de la mera creencia, intuición, dogma, doctrina o fundamentalismos que podrían entorpecer el diálogo, la libertad y la tolerancia(2). Además, es un espacio donde prevalece, como su propio fundamento, una responsabilidad moral y sobre todo ética(3).

El comité de ética se encarga de analizar e interpretar la diversidad de experiencias y perspectivas en la investigación a través del método deliberativo(1), y se apoya en distintos documentos normativos (éticos y legales) con los que intenta resolver, de la manera más prudente y adecuada, los dilemas éticos que se le plantean. Por ello, esta instancia debe constituirse como grupo colegiado, con miembros familiarizados con aspectos básicos de la argumentación y del razonamiento moral, para desempeñar un rol social: acompañar al investigador en la toma de decisiones respecto de los dilemas de orden ético, a través de sugerencias y alternativas para su resolución(1).

El trabajo al interior del comité se sustenta en la justificación racional argumentativa de las acciones preferibles y pertinentes posibles entre varias alternativas(1). El proceso deliberativo es el elemento fundamental y se halla enmarcado en un diálogo multidisciplinario y plural, que permite establecer "las garantías mínimas para identificar los aspectos relevantes por considerar, de modo que la acción práctica, en determinadas circunstancias y en casos complejos, responda a los valores en cuestión" (1:15), que den contenido a los principios, por lo que este objetivo no se consigue con la aplicación rutinaria de principios generales o de normas morales aceptadas.

El ejercicio reflexivo dentro de un comité de ética no se basa en formulas hechas que señalan lo que está bien o mal, o en mandamientos o prohibiciones absolutas; tampoco se reduce exclusivamente a la deontología, es decir a la aplicación de un regla o principio moral; su fin último es garantizar, en la medida de lo posible, un resultado óptimo que atienda a las circunstancias y aspectos particulares de cada caso en el cual es preciso aplicar los principios éticos(4).

El análisis ético que debe realizar el comité se sustenta en la argumentación orientada a identificar el curso de acción más razonable o válido. Esta argumentación puede hacerse con base en los principios éticos, los cuales se consideran guías de actuación y medios para el diálogo; de esta forma, pueden servir de apoyo en el marco de una reflexión individual (autorregulación) o en entornos institucionales (regulación social). En ambos se busca que la acción práctica responda a ciertos valores; así se evita, por un lado, dar un carácter absoluto a los principios, es decir, reconocerlos como válidos ante cualquier circunstancia, y por otro la argumentación moral de rigidez deducti$\mathrm{va}(1)$.

La complejidad del contexto en la investigación provoca que algunos de los principios éticos se contrapongan, por ejemplo, beneficencia y autonomía, por lo que es necesario identificar criterios de aplicación que salvaguarden los valores que dan contenido a estos principios(1). Lo importante es el correcto discernimiento que fundamente "la realidad, en una justa apreciación del contexto institucional, profesional e individual" (5:94).

Lo anterior es una visión panorámica de la razón esencial del comité de revisión ética. Sin embargo, en la práctica, realización o puesta en marcha de estas instancias, la argumentación ética en el análisis de los proyectos de investigación parece insuficiente, debido a que, además de los elementos éticos, los discursos o las resoluciones están impregnados de otros aspectos circundantes a los comités de ética que pueden dificultar su deliberación moral.

El propósito de este manuscrito es analizar los elementos circunscritos al proceso interno de los comités de ética que dificultan su razonamiento deliberativo; lo que a su vez conlleva a distorsionar el ejercicio ético en la investigación e impide el consenso social e institucional sobre ética. A continuación se abordan los siguientes elementos circundantes: la ausencia de la deliberación desde el saber ético; la tensión entre el marco ético y el legal; las particularidades del campo de la salud 
dentro de discernimiento ético y la consideración de los comités como grupos.

\section{Deliberación desde el saber ético}

Una de las críticas más frecuentes y graves que se hace a los comités de evaluación ética es que las decisiones o dictámenes que emiten son resultado de un conjunto de "opiniones" o "creencias" morales personales, o derivadas del ejercicio profesional de cada miembro, y que distan mucho del consenso logrado a través de la cavilación ética, que es la acción esperable. El equívoco de esta situación es que el resultado no sea producto del saber ético, sino dóxico(6,7). La opinión en el trabajo de algunos comités sirve únicamente para la identificación de los dilemas o conflictos éticos en un protocolo científico, pero no para su análisis ni resolución, mucho menos para la toma de decisiones éticas.

Caso aparte es el discurso científico de cada disciplina a que pertenecen los miembros del comité; aunque resulta inseparable de la revisión ética, igual que la opinión, las decisiones que emite un comité de ética no debieran sustentarse en el conocimiento epistémico(7), sino en el saber ético.

En este sentido, entendemos que lo esperable es que la "creencia" y la ciencia queden supeditadas a la deliberación crítica en todo trabajo de los comités. Bajo este supuesto, la primera se ha tratado de ocultar o negar; la segunda ha sido usada de manera predominante y errónea como argumento en las decisiones, lo cual ha debilitado enormemente la acción práctica, razonada a partir de valores y principios éticos de una realidad concreta.

El problema radica, como se mencionó, en que el conocimiento disciplinar de cada miembro de algunos comités, cuando no la mera creencia, prima en la discusión y en la resolución de casos en los comités de ética, lo que conlleva a un detrimento del ejercicio reflexivo de dilemas y la aplicación razonada de principios, y a un debilitamiento de la figura institucional legitimadora de estos comités. Ante ello surgen los siguientes cuestionamientos: ¡es la falta de conocimiento en el campo de la ética o la bioética lo que provoca este conflicto? ¿Hasta qué punto debe estar supeditado el conocimiento científico al análisis ético?
Los miembros de los comités deben estar mínimamente familiarizados con el razonamiento ético y su argumentación, de lo contrario, las decisiones que tomen respecto de los dilemas serán sugerencias o resoluciones que pueda hacer cualquier profesional o comité dedicado a la investigación.

\section{Del marco ético al marco legal}

La aplicación de los requerimientos legales y/o los procedimientos institucionales no siempre se conjunta en la práctica con las recomendaciones éticas. En la mayoría de los casos es primordial hacer un balance claro de estos tres aspectos, imprescindibles para un análisis de elementos circundantes a los comités de evaluación ética.

Conocemos, por un lado, las normas éticas (como el Código de Nuremberg, las actualizaciones de la Declaración de Helsinki, el Informe Belmont, etc.) (1)y, por otro, tenemos normas legales (por ejemplo, en México, la Ley General de Salud, el Reglamento de la Comisión Federal para la Protección contra Riesgos Sanitarios, etc.)(8). Pero, ¿qué pasa cuando, al aplicar algunas de estas normativas en una situación específica, se plantea un conflicto o cuando el establecimiento de procedimientos y guías prácticas para normar la investigación, "impuestas" como una obligación en el actuar del investigador, tienen la función de prevenir ciertas situaciones adversas en las intervenciones, más no de promover la reflexión que esto debiera implicar?

Lo anterior se ejemplifica cuando un médico o investigador puede obtener el consentimiento informado (CI) acatando las reglas institucionales o legales, pero desconociendo el riguroso estándar del principio de autonomía que sustenta tal aplicación ética(8).

Si bien las funciones de los comités de ética en la investigación están estipuladas en reglamentos y certificadas por normas institucionales, esto no es motivo para que en los comités se cavile sobre cuándo y cómo se debe aplicar el reglamento de investigación y las demás disposiciones en cada caso en particular. Vale decir, "se deberían evaluar las reglas institucionales no solo en términos de respeto por la autonomía, sino también en cuanto a las consecuencias de imponer requerimien- 
tos gravosos sobre las instituciones y los profesionales" (8:120). De esta forma, el axioma de la elección por el principio de autonomía (para el consentimiento informado) "debería servir como punto de referencia para una ética adecuada a las reglas institucionales" (8:120).

El problema aparece cuando en investigación se presentan algunos casos en los que es necesario reflexionar desde el marco ético acerca de la aplicación de los procedimientos institucionales o los requerimientos legales, no porque éstos no se respeten o no se cumplan, sino porque en ocasiones es indispensable subrogarlos para actuar de manera ética, es decir, priorizando el respeto a los sujetos de investigación. Por ejemplo, en México, el Reglamento de la Ley General de Salud en materia de investigación(9) establece que el consentimiento informado debe indicar "los nombres y direcciones de dos testigos y la relación que éstos tengan con el sujeto de investigación"; no obstante, el cumplimiento de esta disposición oficial podría provocar perjuicio, dańo o riesgos graves en algunas circunstancias del ámbito de la salud mental, o bien de la investigación de tipo social, debido a que, al trabajar con temas sensibles, como las adicciones o la violencia, la inclusión de los testigos podría interpretarse como una intrusión a la intimidad, la confidencialidad o la privacidad del participante.

Las políticas institucionales pueden representar legítimamente lo justo y lo razonable para los requerimientos de los investigadores. Por ello, la revisión y consultoría que los comités hagan de ellas pueden resultar en grandes beneficios para la institución, esto debido a que la formulación de políticas se hace entonces con una base más reflexiva, lo cual evitaría destinar los casos a los abogados. Este tipo de contribuciones requieren un comité de ética sofisticado, que a su vez dependa de una inversión institucional inicial para la formación de sus miembros(10).

Asimismo, los comités de ética en investigación deberían ayudar a desarrollar guías prácticas y manuales de procedimiento interno para sus instituciones, bajo su contexto particular; por ejemplo, reconocer los riesgos y beneficios de los diferentes tipos de investigación, incluso responder ciertos cuestionamientos más complejos como: ¿en qué situaciones o casos deben predominar los criterios éticos sobre los legales?

\section{Particularidades del campo de la salud dentro del discernimiento ético}

El contexto en que trabajan los comités de ética pocas veces ha sido reconocido como parte del razonamiento deliberativo que realizan sus miembros. Por ejemplo, un comité de evaluación ética que se desenvuelve en el contexto de la salud mental prestará mayor atención a ciertos aspectos éticos, médicos, sociales y legales (como la incapacidad mental, la legislación sobre discapacidad o los derechos de los enfermos mentales, o la lucha contra la discriminación o el estigma para quien padece un trastorno mental), distintos de los de un comité cuyo contexto es la medicina genómica (la confidencialidad y la privacidad de la información, la legislación en la regulación de muestras y tejidos biológicos y la creación de biobancos, etc.).

A pesar de que el marco ético que fundamenta la creación del comité aspira a la universalidad, sería erróneo pensar que todos los comités de evaluación ética son homogéneos. ¿Acaso éstos se enfrentan a los mismos dilemas? Quizá sí, en una proporción considerable, pero cada uno se inscribe en un contexto peculiar que caracteriza, a su vez, el tipo de protocolo de investigación que puede analizar. Entonces ¿̨cuál es la importancia que tiene el contexto de estos comités?

En ocasiones, el contexto de los comités es abordado reduciéndolo o simplificándolo como dilema ético(4). Por ejemplo, algunas especificidades del ámbito de la salud mental, como el estigma, la vulnerabilidad o la incapacidad mental, se han considerado actualmente como conflictos éticos: podemos decir que un paciente con brote psicótico está incapacitado mentalmente para tomar decisiones sobre su salud, ahí no hay dilema. Éste se presenta cuando, en nombre de la investigación científica, se atropellan la dignidad o los derechos humanos del participante(11).

Otro caso se presenta cuando algunas investigaciones sociales se realizan en comunidades o poblaciones consideradas vulnerables; a simple vista, esto no representa un conflicto, pero éste aparece 
cuando los resultados de dichas pesquisas damnifican aún más a estos grupos.

Incluir el contexto en que trabajan los comités dentro de su propio discernimiento ético tal vez permita cavilar sobre cuestionamientos como: ¿dónde empieza la problematización ética de los conceptos asociados con el campo de acción? ¿Cuándo se convierten estos conceptos en dilemas éticos? ¿Cómo tomar decisiones éticas sustentadas en las particularidades de un contexto específico? Y entones, ¿¿cuáles son éstas? En este sentido, se podría dar mayor sustentabilidad a los argumentos y deliberaciones que emiten este tipo de comités a la comunidad a la que pertenecen.

De igual forma, desde el contexto peculiar de los comités, es necesario razonar los conflictos éticos que ahí se presenten, debido a la vaguedad que se tiene acerca de los dilemas específicos que se enfrentan dentro del quehacer de la investigación y las posibles formas de reflexión sobre los mismos. Por ejemplo, de manera somera se sabe que en investigaciones de tipo psicológico o conductual y social los investigadores retienen información en el consentimiento informado para asegurar la validez del estudio, incluso se hace uso del engaño o se informa de manera engańosa a los participantes para estudiar su comportamiento en escenarios "naturales". Los investigadores y comités de evaluación ética debieran estar conscientes de que engañar a los sujetos de investigación puede perjudicarlos o dańarlos(12), por lo que es imprescindible plantear: ¿cuáles serán los criterios o pautas para revisar y aprobar las propuestas que planteen engañar a los participantes? Así como: ¿cuáles derechos de los sujetos(12) prevalecerán por encima del derecho a ser informado y de recibir respuestas honestas a las preguntas sobre su participación en la pesquisa?

Otro conflicto ético en el ámbito de la salud es la posibilidad de intimidación e influencia indebida. Los sujetos potenciales para participar en la investigación son, a la vez, pacientes que reciben atención clínica del médico/investigador, quien, al invitar al paciente a colaborar en un protocolo, puede ejercer una influencia indebida, ya que este último deposita en su médico credibilidad y confianza, y puede asociar que, si se niega a participar, podría dañar la relación o atención terapéu- tica. Sabemos que "el límite entre la persuasión justificable y la influencia indebida es impreciso" (12:47), entonces, ¿qué hacen los comités de evaluación ética ante tal dilema?

El contexto de la salud en el que se inscriben los comités de evaluación ética es lo que determina la particularidad de su análisis. Como se mencionó, la tarea de todos los comités es la reflexión ética, que debería ir más allá de cualquier contexto; sin embargo, las especificidades de este contexto pueden llegar a influenciar el trabajo que realizan los comités, e incluso a obstaculizarlo.

\section{Los comités de evaluación ética como grupos}

En los inicios, cuando los comités de ética emitían sus sugerencias, éstas eran consideradas como una acusación a la moral personal de quien las recibía. Actualmente, los investigadores más jóvenes parecen aceptar estas instancias como guía moral (10). En cuestiones de popularidad - dice Moreno(10)—, los comités podrían brindar la oportunidad para prescribir responsabilidades de las decisiones que originan ambigüedades y que pueden provocar ansiedad a los investigadores.

El inconveniente está en que "los comités de ética se enfrentan al reto de lograr un consenso interno sobre los problemas que enfrentan. El hecho de que los comités estén sujetos a los procesos de grupo pequeño ha sido la fuente de muchas críticas, ya que las relaciones interpersonales en cualquier grupo pueden distorsionar lo que deberían ser deliberaciones"(10:581).

Cualquier decisión ética estará influenciada por la dinámica del grupo de trabajo, esto es, el conjunto de fuerzas sociales y personales que se movilizan en la exposición y la confrontación. La versación diferente de los miembros del comité - dice Lolas - puede llegar a ser una fuente de diversidad que debilite o fortalezca, según la naturaleza del entorno institucional. Ante esto, es relevante que los procesos de los comités sean objeto de estudio, a fin de saber cómo se resuelven los desacuerdos dentro de los mismos y en qué condiciones se puede decir que el grupo colegiado ha llegado al fin de sus deliberaciones sobre un asunto en particular. 
Para Moreno(10), la mayoría de los miembros de los comités de evaluación ética parecen sentirse incómodos cuando deben argumentar explícitamente sobre cuestiones de fondo; de esta manera, expone el autor, las cuestiones morales no se resolverán mediante el recurso de los procedimientos reglamentarios habituales.

Es relevante debatir el "papel del sentimiento, de la razón, de los argumentos y de las relaciones"(5:95), como fenómenos inherentes a los procesos del grupo que constituyen un comité de evaluación ética. "La práctica bioética exige especiales habilidades interpersonales, que se ponen a prueba en la mediación que supone el trabajo mancomunado. Mediación entre personas, entre profesionales, entre instituciones, entre intereses" (5:95).

La deliberación que realizan los comités de ética sobre los proyectos de investigación parece estar contaminada por elementos circundantes a los mismos comités. Esto podría debilitar su presencia, sus expectativas, su labor y sus resultados esperables. Por ello, es necesario estudiar los elementos expuestos, poco comunes, que exploran los confines del propio trabajo interno de los comités(5), con el fin de que sean reconocidos y discutidos en la tarea ética que realizan, en forma permanente y crítica, y de esta manera conseguir el consenso social.

\section{Conclusiones}

Una de las funciones de la bioética es ser un agente para un nuevo consenso social sobre las cuestiones morales en el campo de las ciencias y la tecnología. En este sentido, sus vehículos principales son las comisiones de bioética, que operan nacional e internacionalmente, y los comités de evaluación ética, en un nivel institucional(10).

Los comités de ética son los vehículos más prometedores para la creación de consenso institucional. Los grupos en general bien integrados, con individuos competentes en la materia, son agentes poderosos de cambio para cualquier institución(10). Por lo que también se hace necesario el estudio de su proceso interno.

Si bien los comités de ética se encuentran entre las manifestaciones más visibles de la bioética en el ámbito social orientado a las reformas y al trabajo multidisciplinario, y la aceptación que han recibido estas instancias en los últimos años ha sido amplia, es poco lo que se puede decir acerca de sus funciones y sus metas(10), principalmente porque se ha rezagado el análisis del trabajo o ejercicio interno de los comités: ¿cómo deliberan? ¿En qué casos se pondera el marco legal? ¿Cuáles son los dilemas particulares al ámbito de inserción de los comités? ¿Cuáles los elementos del proceso grupal que permiten la constitución del comité? Entre otras.

La deliberación ética es un elemento que, por lo general, se presupone como función y método del trabajo de un comité; no obstante, en la evaluación de protocolos de investigación lo imperante son debates y resoluciones sustentados en el conocimiento disciplinar o en juicios morales de los miembros de algunos comités. Esto obstaculiza la cavilación ética de las pesquisas y también ocasiona un debilitamiento en la legitimación institucional de dichas instancias, pero principalmente desestima el ejercicio ético.

Las consecuencias de que los comités no realicen un análisis ético de los proyectos científicos son claramente conocidas, no así las posibles enmiendas. La falta de preparación o educación formal sobre ética en investigación o bioética de los miembros de estos grupos es la razón más citada como origen de este problema. La profesionalización de los integrantes del comité ha sido una alternativa para corregir este conflicto, sin embargo, representa otra serie de implicaciones, incluso éticas, actualmente difíciles de sortear(13).

Por lo menos, los miembros de los comités de ética en investigación deben tener conocimientos básico sobre ética o bioética, "amplio panorama que incluya un marco de valores y perseguir una educación continua, y legitimarse a través de la autonomía profesional" (13:12) para, de esta forma, diferenciarse de otros profesionales o comités dedicados a la investigación. Tal vez son ideales a seguir, pero mientras no se aborden sistemáticamente los procesos internos de los comités de ética — que incluyan la operacionalidad de sus funciones, la supedición del conocimiento científico al análisis ético, entre otros-, se continua- 
rá distorsionando la argumentación razonada en materia de ética e investigación.

Conocer cómo distender las situaciones que contraponen el marco ético al marco legal es un aspecto que se ha mitigado y por lo tanto no se ha indagado. La acción que ha contrarrestado el conflicto entre lo ético y legal es la aplicación de una ética en su carácter más normativo, la cual prescribe normas en la investigación supeditadas a las políticas o reglas institucionales, obligatorias para el investigador, y que en la mejor de las intenciones tratan de evitar situaciones desfavorables en las pesquisas, pero que no generan la reflexión que debieran originar.

Dado lo anterior, es necesario que los comités de ética incluyan en su trabajo interno el análisis de las circunstancias que priorizan los criterios éticos y los legales. Los comités tienen una función educativa, además de la mera revisión y evaluación de proyectos de investigación, que podrán ejercitar a través de generar información orientadora al respecto, dentro del contexto particular de la institución perteneciente.

La importancia de abordar las particularidades del campo de la salud en el que se inscriben los comités de ética se debe a su influencia en la deliberación ética. No solo porque los dilemas dependen de su contexto, sino porque éste puede determinar los argumentos y deliberaciones que expone el comité. Sin una clara conciencia de este elemento innegable se restringe el funcionamiento y metas de los comités de evaluación ética. Incluir el contexto en la reflexión ética del propio comité arrojará mayor eficiencia en el proceso deliberativo.

El comité de ética es un grupo cuyas relaciones interpersonales están impregnadas de fuerzas sociales y personales que se movilizan en la exposición y la confrontación, lo cual facilita una influencia directa en el diálogo ético imperante en el trabajo del comité y dificulta en ocasiones el consenso, como producto final de la deliberación ética de estos grupos. Ni siquiera una normativa ética, ya sea código o guía, podría evitar la dinámica inherente a cualquier conformación grupal. Dar cabida al entendimiento de las emociones, vínculos sociales, posiciones de poder o estatus no obedece a psicologizar el razonamiento ético, sino a contextualizar este razonamiento con el fin de ubicarlo y no neutralizar la vida de un grupo colegiado.

Lolas menciona que, "indirectamente, los comités de ética de la investigación ejercen un efecto benéfico sobre las instituciones, al brindar a sus miembros y sus usuarios la certidumbre de que los conflictos pueden expresarse, estudiarse, resolverse o disolverse (...) (además, son una forma) de conciencia reflexiva para la institución que los alberga, permitiendo sus naturales ambivalencias, sus internas discrepancias y su pluralidad de intereses, y reforzando sus metas y objetivos"(5:97). Por ello, la reflexión final apunta a advertir que existen elementos circunscritos al proceso interno de los comités de evaluación ética, como la ausencia de la deliberación desde el saber ético, la tensión entre el marco ético y el legal, las particularidades del campo de la salud dentro del discernimiento ético y la consideración de los comités como grupos, que dificultan su razonamiento deliberativo.

La discusión sobre los elementos presentados debe integrarse al trabajo autorregulativo de los propios comités de ética; de lo contrario, estas instancias tergiversarán el ejercicio ético en la investigación e impedirán el consenso social e institucional sobre ética.

\section{Agradecimientos}

Este trabajo fue posible gracias al proyecto "La aplicación de los principios bioéticos en la atención clínica y la prevención del intento de suicidio", del Instituto Nacional de Psiquiatría Ramón de la Fuente INPRFM-4601. Financiamiento por el Consejo Nacional de Ciencia y Tecnología (CONACyT-91082). 
Elementos circundantes a los comités de ética que dificultan su razonamiento deliberativo - Liliana Mondragón

Referencias

1. Comisión Nacional de Bioética. Guía Nacional para la Integración y Funcionamiento de Comités de Ética en Investigación. México: Comisión Nacional de Bioética, Secretaría de Salud; 2010.

2. Kottow M. Introducción a la Bioética. Santiago de Chile: Mediterráneo; 2005.

3. Mondragón L. Ética de la investigación psicosocial. Salud Mental 2007; 30: 25-31.

4. Montuschi L. Ética y razonamiento moral. Dilemas morales y comportamiento ético en las organizaciones. Universidad de CEMA. Feb. 2004. Citado en febrero 13, 2011. Disponible en http://www.biblioteca.org.ar/zip3.asp?texto=550219

5. Lolas F. Bioética. El diálogo moral en las ciencias de la vida. 2a ed. Santiago de Chile: Mediterráneo; 2003.

6. Ferrater Mora J. Diccionario de Filosofía. Barcelona: Editorial Ariel; 1999: 936.

7. Álvarez J, Lolas F, Outomuro D. Historia de la ética en investigación con seres humanos. En Lolas F, Quezada A, Rodríguez E, (eds.) Investigación en Salud. Dimensión Ética. Santiago de Chile: CIEB, Universidad de Chile; 2006: 39-46.

8. Beauchamp TL, Childress JF. Principles of Biomedical Ethics. $6^{\text {th }}$ edition. New York: Oxford; 2009.

9. Reglamento de la Ley General de Salud en materia de investigación en seres humanos. Diario Oficial de la Federación el 7 de febrero de 1984, citado en febrero 26, 2011. Disponible http://www.salud.gob.mx/unidades/cdi/nom/compi/ rlgsmis.html

10. Moreno J. Ethics Committee and Ethics Consultants. In: Kuhse H, Singer P, (eds.) A Companion to bioethics. $2^{\text {nd }}$ ed. Singapur, Hong Kong: Wiley-Blackwell; 2009: 573-583.

11. Comisión Nacional de los Derechos Humanos. Los Derechos Humanos de las personas con enfermedades mentales. México: $\mathrm{CNDH} ; 2007$.

12. Consejo de Organizaciones Internacionales de las Ciencias Médicas (CIOMS). Pautas Éticas Internacionales para la Investigación Biomédica en Seres Humanos (rev. 2002). Santiago de Chile: Programa Regional de Bioética OPS/OMS; 2003.

13. Sagols L. ¿Profesionalizar la bioética? Bios \& ethos 2011; 3: 6-13.

Recibido: 27 de abril de 2012

Aceptado: 18 de junio de 2012 